\title{
Direct preparation of standard functional interfaces in oxide heterostructures for 2DEG analysis through beam-induced platinum contacts
}

Cite as: Appl. Phys. Lett. 113, 131603 (2018); https://doi.org/10.1063/1.5046093

Submitted: 25 June 2018 . Accepted: 15 September 2018 . Published Online: 28 September 2018

Rafael A. C. Amoresi (D, Leonélio Cichetto, Swarup Kundu (D), Marcio D. Teodoro, Gilmar E. Marques, Elson Longo (D), Juan Andrés, Adenilson J. Chiquito, and Maria A. Zaghete
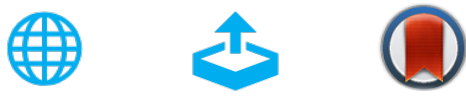

\section{ARTICLES YOU MAY BE INTERESTED IN}

Yttrium zinc tin oxide high voltage thin film transistors

Applied Physics Letters 113, 132101 (2018); https://doi.org/10.1063/1.5048992

Impact of a surface $\mathrm{TiO}_{2}$ atomic sheet on the electronic transport properties of $\mathrm{LaAlO}_{3} / \mathrm{SrTiO}_{3}$ heterointerfaces

Applied Physics Letters 113, 141602 (2018); https://doi.org/10.1063/1.5046876

Investigation of $\mathrm{LaAlO}_{3}-\mathrm{SrTiO}_{3}$ field-effect transistors under hydrostatic pressure Applied Physics Letters 113, 143507 (2018); https://doi.org/10.1063/1.5050322
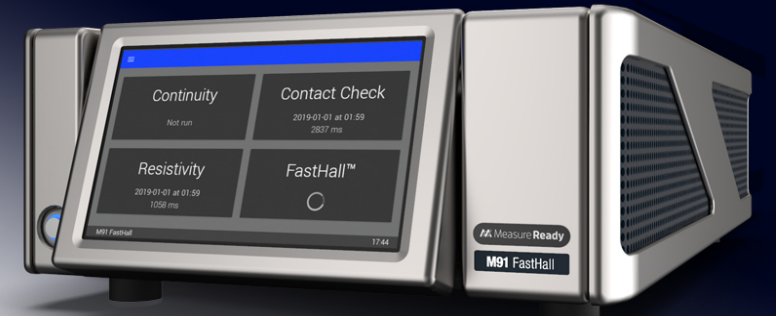

\section{Measure Ready} M91 FastHall ${ }^{\text {TM }}$ Controller

A revolutionary new instrument for complete Hall analysis 


\title{
Direct preparation of standard functional interfaces in oxide heterostructures for 2DEG analysis through beam-induced platinum contacts
}

\author{
Rafael A. C. Amoresi, ${ }^{1, a)}$ Leonélio Cichetto, Jr., ${ }^{2,3}$ Swarup Kundu, ${ }^{1}$ Marcio D. Teodoro, ${ }^{4}$ \\ Gilmar E. Marques, ${ }^{4}$ Elson Longo, ${ }^{2}$ Juan Andrés, ${ }^{3}$ Adenilson J. Chiquito, ${ }^{4}$ \\ and Maria A. Zaghete ${ }^{1}$ \\ ${ }^{1}$ LIEC - Chemistry Institute, São Paulo State University - UNESP, 14800-060 Araraquara, SP, Brazil \\ ${ }^{2}$ LIEC - Department of Chemistry, Universidade Federal de São Carlos-UFSCAR, 13565-905 São Carlos, \\ SP, Brazil \\ ${ }^{3}$ Department of Analytical and Physical Chemistry, University Jaume I (UJI), Castelló 12071, Spain \\ ${ }^{4}$ Department of Physics, Universidade Federal de São Carlos-UFSCAR, 13565-905 São Carlos, SP, Brazil
}

(Received 25 June 2018; accepted 15 September 2018; published online 28 September 2018)

\begin{abstract}
Two-dimensional electron gas (2DEG) in $\mathrm{SrTiO}_{3} / \mathrm{LaAlO}_{3}$ heterostructures has been extensively studied in the last few years; however, little attention has been given to a practical way to contact electrically the low dimensional gas at the interface. This work demonstrates a method to contact the 2DEG formed at the oxide interfaces connected by platinum electrodes which were made by the decomposition of organometallic gas using focused ion beams. On the surface, the electrodes were defined through photolithography, and at the interface, the electrodes were deposited through the focused ion beams and electrons, which were then evaluated. The quality of the interface electrodes was evaluated at two different partial oxygen pressures $\left(\mathrm{pO}_{2}\right)$ used for the film deposition: low $\left(10^{-4} \mathrm{mbar}\right)$ and high $\left(10^{-1} \mathrm{mbar}\right)$. The electrode deposition conditions using electrons or ions have resulted in different rates of metal deposition and interaction with the interface leading to either metallic (2DEG) or insulating behavior. Published by AIP Publishing.

https://doi.org/10.1063/1.5046093
\end{abstract}

High mobility in two-dimensional electron gas (2DEG) has been obtained in epitaxially grown III-V semiconductor devices such as GaAs/AlGaAs heterostructures, and superlattices were grown by high cost synthesis methods such as Molecular Beam Epitaxy (MBE) or vapor deposition techniques such as Metal Organic Chemical Vapor Deposition (MOCVD). ${ }^{1,2}$ Among the most recent discoveries in high electron mobility 2D materials are heterostructured oxides such as $\mathrm{Mg}_{\mathrm{x}} \mathrm{Zn}_{1-\mathrm{x}} \mathrm{O} / \mathrm{ZnO}$ and $\mathrm{SrTiO}_{3} / \mathrm{LaAlO}_{3}$ (STO/LAO). ${ }^{3,4}$ The 2DEG found at the interfaces of STO/LAO heterostructures is of high importance for the development of electronic devices, ${ }^{5}$ superconductors, ${ }^{6}$ and spintronics. ${ }^{7}$ Since the discovery by Hwang and Ohtomo ${ }^{8}$ of an electron gas confined in this heterostructure, numerous works have explored its origin using different perovskites characterized by insulating bands. ${ }^{9-11}$ Among those, the most accepted explanations to the 2DEG observation are the polar catastrophe, ${ }^{12,13}$ the cationic intermixture, ${ }^{14}$ defects due to oxygen vacancies, ${ }^{15}$ or strontium ${ }^{16}$ vacancies.

Electron gas present in the oxide heterostructure interfaces can be obtained by a less complex process such as Pulsed Laser Deposition (PLD), a better cost effective film deposition technique. Also, this technique is useful to develop devices ready for applications and for basic research. However, regarding the construction of a useful device, there are several steps between obtaining the high quality interface and the application itself. Each step from the manufacture of the samples to the transport measurements is of fundamental importance in order to obtain a desired high mobility electron gas and all of the characteristic physical properties of

${ }^{a)}$ E-mail: rafaelciola@yahoo.com.br the system. In the case of the system studied in this work, the STO/LAO heterostructures, there are five processing steps that are critical: (i) the treatment of the substrate surface of $\mathrm{SrTiO}_{3}$, (ii) the setting up of the deposition conditions of the $\mathrm{LaAlO}_{3}$ film such as the partial pressure of oxygen $\left(p \mathrm{O}_{2}\right)$ during deposition, (iii) the sintering conditions after deposition, and finally, (vi) the process of contacting the electron gas at the interface. Regarding the $\mathrm{pO}_{2}$ conditions for LAO film growth on STO, it was noted by Kalabukhov et al. ${ }^{17}$ and also by several other works ${ }^{15,18}$ that a metallic behavior for films deposited at partial oxygen pressures lower than $10^{-3}$ mbar was always obtained. In these samples, the Hall mobility decreased from $10^{4} \mathrm{~cm}^{2} \mathrm{~V}^{-1} \mathrm{~s}^{-1}$ for deposition at $10^{-6}$ mbar $\mathrm{O}_{2}$ to a mobility of $10^{3} \mathrm{~cm}^{2} \mathrm{~V}^{-1} \mathrm{~s}^{-1}$ at $10^{-4}$ mbar, and an insulating behavior was observed for the $\mathrm{pO}_{2}$ below $10^{-2}$ mbar. This is related to the oxygen vacancies created during the deposition process, and it is one of the responsible factors for the conductivity in the STO/LAO heterostructures.

Establishing good electrical contacts to the oxide heterostructure interface where the electron gas is confined determines the reproducibility and reliability of the transport measurements and the efficiency in capturing the interface charge carriers. The first study of this system ${ }^{8}$ regarding the electrical contact behavior has been made by laser-annealed ohmic contacts to reach the interface where the $2 \mathrm{DEG}$ is formed. This contact method produced an array of microcracks on the surface of the LAO films and then exposed the electron gas to the environment causing the loss of the quality of electron transport properties. ${ }^{19}$ In another study, samples have been electrically contacted through the surface using different geometries, and to contact the interface, an ultrasonic wirebonder with aluminum wire was used. ${ }^{20-22}$ 
TABLE I. Growth conditions of the films and method of depositing the electrodes.

\begin{tabular}{lcccc}
\hline \hline Sample & High-IBID & Low-IBID & High-EBID & Low-EBID \\
\hline Deposition $\mathrm{pO}_{2}$ (mbar) & $10^{-1}$ & $10^{-4}$ & $10^{-1}$ & $10^{-4}$ \\
Deposition electrode & IBID & IBID & EBID & EBID \\
\hline \hline
\end{tabular}

By this method, mechanical deformation behavior dominated by the fracture in grain boundaries was observed, ${ }^{23}$ leading to damage of the samples and consequently of their electrical properties. Other possibilities for the electrical contact of the interface are ion or wet-etching. However, these techniques can mask the conductivity and/or degrade the conductivity as already studied. ${ }^{24,25}$ Several techniques use patterning schemes that do not cause degradation of the interface, requiring the deposition of some amorphous films layers and different lithographic steps. ${ }^{19,26}$ In summary, it was observed that surface/interface contact techniques usually have preparation sequences which cause damage to the sample or at least mask its transport properties.

In this letter, we evaluated a protocol for making the contact with the two-dimensional electron gas trapped at the interface of STO/LAO. For this, we deposited the films by the PLD technique in two conditions: one, where the thin films were deposited at low $\mathrm{pO}_{2}$, which commonly has the 2DEG behavior and the other one at a higher $\mathrm{O}_{2}$ pressure that usually has insulating behavior. ${ }^{17}$ Then, we designed a standard electrode pattern on the surface of the film by conventional photolithography and evaluated two innovative procedures for contacting the electron gas at the interface. The deposition of platinum by ions and electrons was performed using focused ion beam (FIB) microscopy. LAO films were grown on $\mathrm{TiO}_{2}$-terminated STO monocrystalline (100) substrates by PLD in a high vacuum chamber, using a $\mathrm{KrF}$ excimer laser with a wavelength of $248 \mathrm{~nm}$ at a repetition rate of $2 \mathrm{~Hz}$ and the laser fluency on the target surface of $\sim 1.8 \mathrm{~J} \mathrm{~cm}^{-2}$, with 240 laser shots ( $\sim 4.5 \mathrm{~nm}$ film thickness). The deposition temperature was $730^{\circ} \mathrm{C}$, and two oxygen partial pressures were used as described in Table I. The substrate-target distance was $40 \mathrm{~mm}$. After LAO growth, the samples were thermally treated at $550{ }^{\circ} \mathrm{C}$ for $60 \mathrm{~min}$ (in situ) at an oxygen partial pressure of 200 mbar.

Figure 1(a) depicts an AFM analysis of the surface morphology of the film, with a smooth and regular surface, showing a quadratic roughness of $0.4 \mathrm{~nm}$. Between the homogeneous regions of the film, steps are formed, due to the previous treatment performed on the surface of the substrate. ${ }^{27}$ On the top of this surface morphology type, the contact patterns for the surface and interface were built to evaluate the 2DEG. For this purpose, the conventional photolithography technique was used for making surface contacts. The contacts consisted of $15 \mathrm{~nm}$ of $\mathrm{Ti}$ followed by $80 \mathrm{~nm}$ of $\mathrm{Au}$, thus forming the surface electrode [Figs. 1(b) and $1(\mathrm{c})]$. The entire procedure was performed in a clean

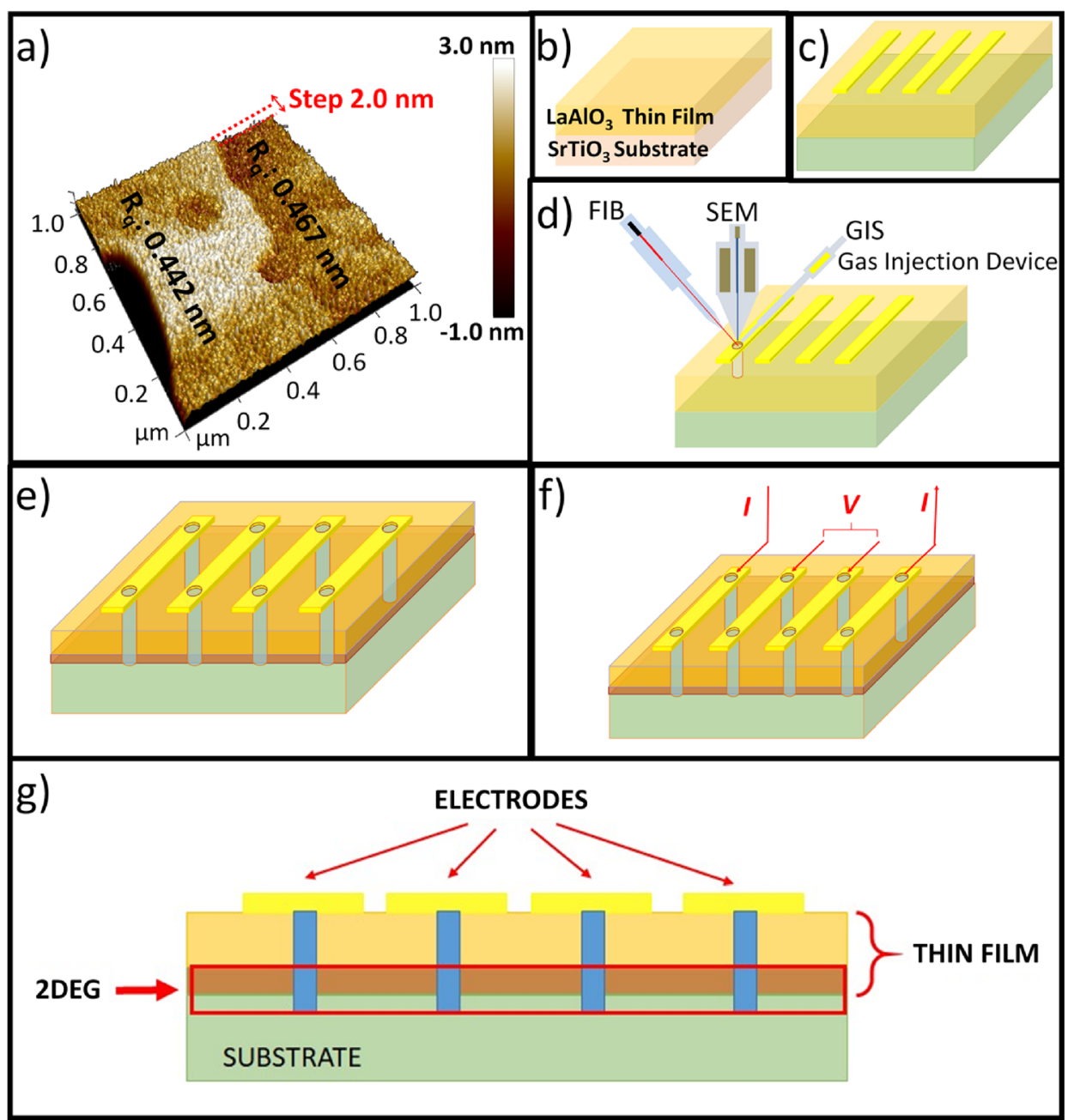

FIG. 1. (a) AFM image of the surface of the film obtained by PLD and (b)-(g) illustrative diagram of the surface and interface contacts made for analysis of the electric transport properties. 
room. For the interface contacts, the electrodes were made through a FIB microscope (FEI Helios Nanolab 600I). Using this procedure, we were able to drill holes of $400 \mathrm{~nm}$ in diameter and $250 \mathrm{~nm}$ in depth in samples. This depth was deep enough to overcome the surface electrode, the LAO film, and the top surface of the STO substrate and reach the 2DEG, as seen in Figs. 1(d) and 1(e). The holes were made using the gallium ion beam (at $8 \mathrm{kV}$ and a current of $0.11 \mathrm{nA}$ ). Then, inside the holes, platinum ions were deposited forming a Pt electrode which was in contact with the two-dimensional electron gas as seen in Figs. 1(f) and 1(g). The whole electrode deposition process was investigated using two configurations of the FIB system: EBID_Electron Beam-Ion Deposition in the 10kV@0.17nA configuration; and IBID_-Ion Beam-Ion Deposition using 16 kV@0.13nA. The procedure for preparing the interface electrodes was performed with few steps and avoided exposing the interface containing the $2 \mathrm{DEG}$ to the environment.

The scanning electron microscopy images shown in Fig. 2 illustrate the steps described in Fig. 1. Figure 2(a) depicts the surface electrodes deposited by the lithographic process, consisting of $10 \mu \mathrm{m}$ wide parallel bars and separated by an equal distance from each other. Figures 2(b) and 2(c) show the surface electrodes after being drilled through the FIB microscope. It is possible to observe from these images a homogeneous pattern of holes [Fig. 2(b)], showing the reproducibility of transport measurements in the sample. It is also important to verify that it is possible to realize the hole and electrode deposition at a specific site of the film surface for electrical characterization, due to the characteristics of the FIB. It is important to take into consideration the terraces, ledges, and steps formed on the surface of the STO substrate after removal of atomic layers of $\mathrm{SrO}^{27}$ to obtain the Ti-O termination. This is a crucial step for obtaining the 2DEG at the interface of the STO/LAO heterostructure. ${ }^{28}$
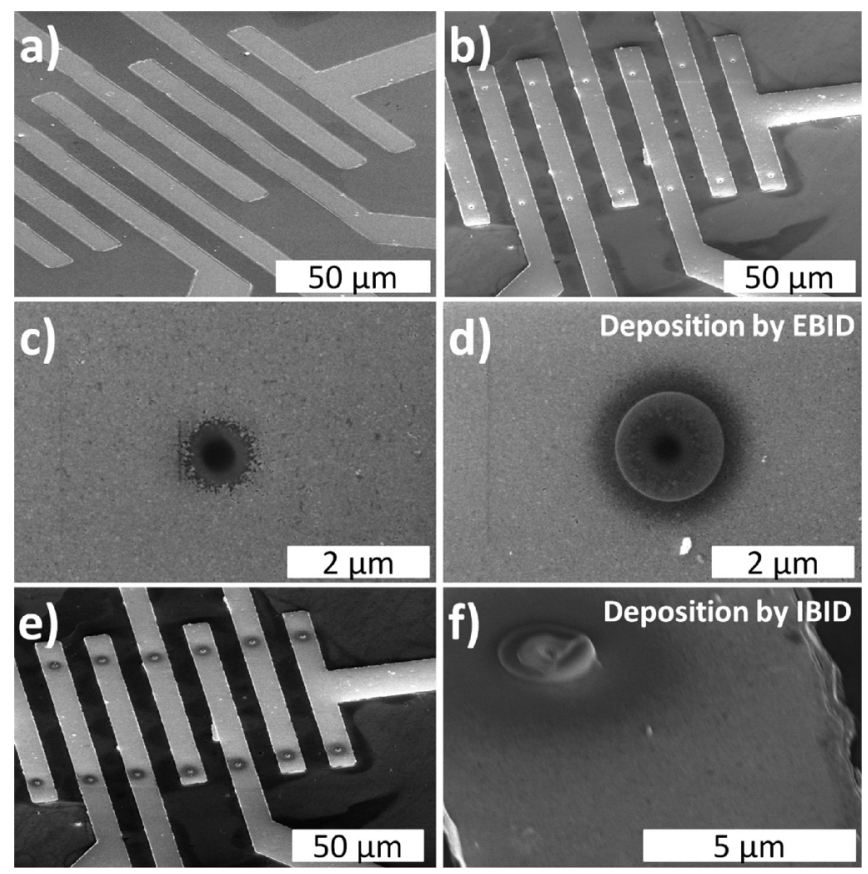

FIG. 2. (a) SEM image of electrodes deposited by lithography before drilling, (b) and (c) after holes are made by FIB, and after deposition of platinum by (d) EBID and (e) and (f) IBID.
Figures 2(d)-2(f) display the surface of the electrodes, after deposition of Pt in the holes, by EBID and IBID techniques, respectively. For both used techniques, the complete filling of the holes was observed. The beam induced deposition techniques are characterized by a process whereby a solid material can be deposited inside a solid substrate by means of the primary beam of electrons or ions, through decomposition of a molecular precursor. In this process, a vapor precursor is introduced into the substrate and adsorbed onto the surface site. That site is exposed to a beam of electrons or ions, inducing dissociation reactions and resulting in a deposited solid and a volatile by-product. ${ }^{29}$ Comparing Fig. 2(d) with Fig. 2(f), the electrodes obtained from IBID are denser than those deposited by EBID, which is likely due to the high deposition rate. ${ }^{30}$ This results in changes of the resistivity of deposited electrodes by EBID and IBID. Previous studies showed that the resistance of electrodes deposited by ions is usually lower. $^{30,31}$ Our results demonstrate that the photolithographic process, coupled with the technique of focused beam microscopy, allowed us to draw any geometry along the surface of the films. Additionally, it is also possible to make the electrodes along the direction of the steps formed on the surface of the film. This is interesting for local analysis in the sample without damaging the surface.

In order to evaluate the efficiency of the techniques used in the preparation of the electrodes, electrical transport measurements were performed and the results are shown in Fig. 3. Four systems were evaluated by varying the $p \mathrm{O}_{2}$ in deposition and the method used for achieving the electrical contacts, as described in Table I. The temperature-resistance analysis (Fig. 3) was carried out at different temperatures from 10 to $300 \mathrm{~K}$ using a closed-cycle helium cryostat (Janis ${ }$, model CCS150). The resistance was obtained using common dc techniques (Keithley®, model 2400C), and for high resistance measurements, an electrometer (Keithley ${ }^{\circledR}$, model 6517B) was used. Figures 3(a) and 3(b) show the resistance of the system with the interface electrode obtained by IBID. For the system deposited at high $\mathrm{pO}_{2}$ [Fig. 3(a)], the electrical resistance over the entire temperature range $(10-300 \mathrm{~K})$ is very high $\left(10^{19}-10^{20} \Omega\right)$. For the system deposited at low $\mathrm{pO}_{2}$ [Fig. 3(b)], the electrical resistance is of the order of $10^{11} \Omega$ at $10 \mathrm{~K}$ and decreases approximately two orders of magnitude with increasing temperature. In both cases for the films with the electrodes made by IBID, no metallic behavior was observed, which would infer the presence of 2DEG. Clearly, the system with interface electrodes obtained by EBID [Figs. 3(c) and 3(d)] showed distinct behavior from those obtained by IBID. In Fig. 3(c), for the films obtained at high $\mathrm{pO}_{2}$, the behavior is also insulating; however, these have lower electrical resistance $\left(10^{8} \Omega\right)$. At low deposition pressures [Fig. 3(d)], the films presented a metallic behavior-increase in the resistance as the temperature increases - which can be considered a clear evidence of the presence of a highly conducting two-dimensional electron gas between the STO/LAO insulating perovskites. The inset in the figure shows a current-voltage characterization with a linear relationship between the applied voltage and current, indicating an ohmic behavior. The density of carriers and mobility for this sample were found to be 


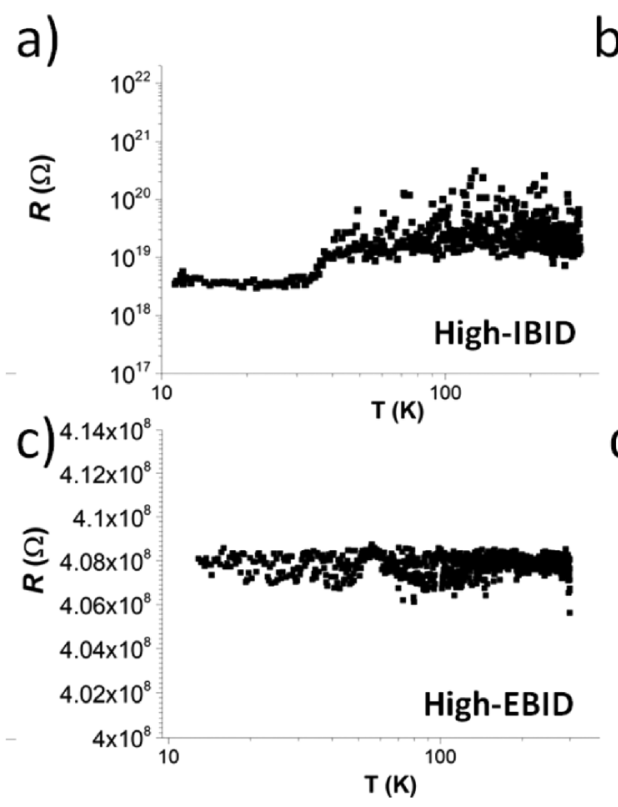

b)

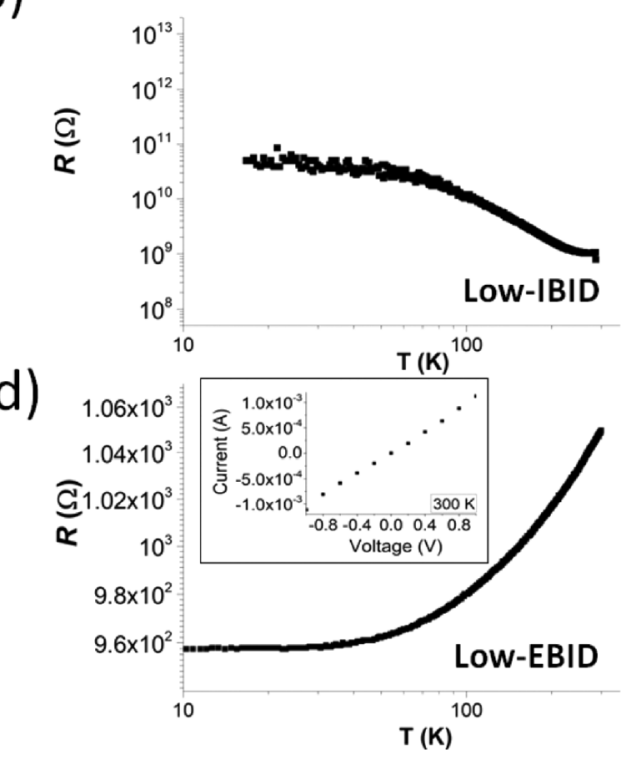

FIG. 3. Temperature-dependent resistance at different $\mathrm{pO}_{2}$ (high and low) of films using (a) and (b) IBID and (c) and (d) EBID interface electrodes, respectively. The inset shows currentvoltage characterization for the lowEBID sample.
$2.63 \times 10^{12} \mathrm{~cm}^{-2}$ and $276 \mathrm{~cm}^{2} \mathrm{~V}^{-1} \mathrm{~s}^{-1}$, respectively. These values are in agreement with those published earlier in the literature. ${ }^{4,32}$ Thus, by using the method presented here for obtaining both 2DEG and electrical contacts, we have found devices (EBID) which are potentially useful for practical applications (resistance $\sim \mathrm{k} \Omega$ ).

Regarding the deposition conditions of the films, as expected and previously explained above, the systems obtained at high $\mathrm{pO}_{2}$ have insulating behaviors due to the dependence of the resistance with the structural formation of the film, such as the absence of oxygen vacancies in the structure. ${ }^{15,17,18}$ Two tests with different deposition pressures were performed precisely to evaluate whether the interface electrodes would have the capacity to differentiate the electric response of different structures formed at the interface. Concerning the interface electrodes, one would expect metallic behavior for the system obtained at low deposition pressure by IBID, because the electrodes were denser as observed in the micrograph analysis and therefore a greater amount of gas was converted to the platinum in the deposition. ${ }^{30}$ However, only the electrodes obtained through the EBID technique proved to be effective in detecting the two-dimensional electron gas and to analyze the variation of the deposition conditions. Previous studies of metallic patterns deposited by IBID and EBID showed that when performing halos of different distances within these patterns, the contacts obtained by ions had lower resistance. ${ }^{31}$ The lower resistance was the result of the presence of defects that originated during the deposition. The IBID technique causes damage to the surfaces which are exposed to the ions because of the high energy $\mathrm{Ga}^{+}$ions used during this procedure. ${ }^{33}$ In our case, this is decisive for analysis of the 2DEG.

Based on the above results, we can propose a simple model described by the following equations: Eq. (1) represents the interface formation process of 2DEG; when the focused beam (used for contacts definition) interacts with the interface region where the $2 \mathrm{DEG}$ is present, there is another kind of interaction as described by Eqs. (2)-(3)

$$
\begin{gathered}
M O_{y} \rightarrow M O_{y-1} \cdot V_{O}^{x} \rightarrow M O_{y-1}^{\prime \prime} \cdot V_{O}^{\cdot} \\
M O_{y-1} \cdot V_{O}^{x} \stackrel{2 G a^{+}}{\longrightarrow} M O_{y-1} \cdot V_{O}^{\cdot}+2 G a^{x} \\
M O_{y-1} \cdot V_{O}^{x} \stackrel{e^{-}}{\longrightarrow} M O_{y-1}^{\prime \prime} \cdot V_{O}^{*}+e^{-}
\end{gathered}
$$

In these equations, $\mathrm{MO}_{\mathrm{y}}$ is a metal oxide, $\mathrm{V}_{\mathrm{O}}$ is an oxygen vacancy, and the superscripts $\mathrm{x}$ and $\cdot \cdot$ represent the neutral and doubly ionized charges, respectively. The symbol " represents a pair of electrons. Equation (2) describes the interface contact through the deposition of an electrode by using ions (IBID), in which the interaction of electrons at the interface with the ions avoids the observation of the 2DEG. Equation (3) describes the interface contact through the deposition of the electrode by electrons, in which there is no change in the 2DEG which could be caused by the definition of the electrode contacts (as it was seen in the IBID technique).

In this article, we demonstrate a method of fabricating interface contact to analyze the 2DEG between oxide heterostructures. We evaluated the two possibilities of Pt deposition using ion beams through IBID and EBID. The IBID technique masked the electrical properties of the interface proving not to be useful for the analysis of the two-dimensional electron gas. By using EBID, it was possible to detect the presence of the electron gas. This technique does not lead to exposure of the electron gas to the environment and fissures or cracks on the sample's surfaces and does not require many processing steps and sequences using amorphous films and lithographic processes.

The authors acknowledge the Grant CEPID/CDMF-São Paulo Research Foundation-FAPESP (Proc. Nos. 2013/072962, 2014/01371-5, 2017/23663-6, and 2017/13024-6) and the National Council for Scientific and Technological Development $(\mathrm{CNPq})$ for the financial support granted in the course of this research. J.A. acknowledges financial support from the Generalitat Valenciana for Prometeo/2016/079, the Spanish MINECO for Project No. CTQ2015-65207-P, and Universitat Jaume I, Project No. UJI-B2016-25. We are also grateful to the LMA-IQ for providing the FEG-FIB-SEM facilities. 
${ }^{1}$ K. K. Choi, D. C. Tsui, and S. C. Palmateer, Phys. Rev. B 32, 5540 (1985).

${ }^{2}$ F. W. Smith, A. R. Calawa, C. L. Chen, M. J. Manfra, and L. J. Mahoney, IEEE Electron Device Lett. 9, 77-80 (1988).

${ }^{3}$ Y. Kozuka, A. Tsukazaki, D. Maryenko, J. Falson, S. Akasaka, K. Nakahara, S. Nakamura, S. Awaji, K. Ueno, and M. Kawasaki, Phys. Rev. B 84, 033304 (2011).

${ }^{4}$ H. Y. Hwanga, A. Ohtomo, N. Nakagawa, D. A. Muller, and J. L. Grazul, Physica E 22, 712-716 (2004).

${ }^{5}$ H. Liang, L. Cheng, X. Zhai, N. Pan, H. Guo, J. Zhao, H. Zhang, L. Li, X. Zhang, X. Wang, C. Zeng, Z. Zhang, and J. G. Hou, Sci. Rep. 3, 1975 (2013).

${ }^{6}$ M. Yang, J. Zhou, T. C. Asmara, P. Krüger, X. J. Yu, X. Wang, C. Sanchez-Hanke, Y. P. Feng, T. Venkatesan, and A. Rusydi, ACS Appl. Mater. Interfaces 10, 9774 (2018).

${ }^{7}$ E. Lesne, Y. Fu, S. Oyarzun, J. C. Rojas-Sánchez, D. C. Vaz, H. Naganuma, G. Sicoli, J.-P. Attané, M. Jamet, E. Jacquet, J.-M. George, A. Barthélémy, H. Jaffrès, A. Fert, M. Bibes, and L. Vila, Nat. Mater. 15, 1261 (2016).

${ }^{8}$ A. Ohtomo and H. Y. Hwang, Nature 427, 423 (2004).

${ }^{9}$ P. Moetakef, C. A. Jackson, J. Hwang, L. Balents, S. J. Allen, and S. Stemmer, Phys. Rev. B 86, 201102 (2012).

${ }^{10}$ R. Ghosh, D. Basak, and S. Fujihara, J. Appl. Phys. 96, 2689 (2004).

${ }^{11}$ A. Aezami, M. Abolhassani, and M. Elahi, J. Alloys Compd. 587, 778 (2014).

${ }^{12}$ N. Nakagawa, H. Y. Hwang, and D. A. Muller, Nat. Mater. 5, 204 (2006).

${ }^{13}$ A. Savoia, D. Paparo, P. Perna, Z. Ristic, M. Salluzzo, F. M. Granozio, U. S. di Uccio, C. Richter, S. Thiel, J. Mannhart, and L. Marrucci, Phys. Rev. B 80, 075110 (2009).

${ }^{14}$ L. Qiao, T. C. Droubay, T. Varga, M. E. Bowden, V. Shutthanandan, Z. Zhu, T. C. Kaspar, and S. A. Chambers, Surf. Sci. 605, 1381-1387 (2011).

${ }^{15}$ A. Brinkman, M. Huijben, M. Van Zalk, J. Huijben, U. Zeitler, J. C. Maan, W. G. Van Der Wiel, G. Rijnders, D. H. A. Blank, and H. Hilgenkamp, Nat. Mater. 6, 493 (2007).

${ }^{16}$ F. Gunkel, S. Wicklein, S. Hoffmann-Eifert, P. Meuffels, P. Brinks, M. Huijben, G. Rijnders, R. Waser, and R. Dittmann, Nanoscale 7, 1013-1022 (2015).
${ }^{17}$ A. Kalabukhov, R. Gunnarsson, J. Börjesson, E. Olsson, T. Claeson, and D. Winkler, Phys. Rev. B 75, 121404 (2007).

${ }^{18}$ W. Siemons, G. Koster, H. Yamamoto, W. A. Harrison, G. Lucovsky, T. H. Geballe, D. H. A. Blank, and M. R. Beasley, Phys. Rev. Lett. 98, 196802 (2007).

${ }^{19}$ C. W. Schneider, S. Thiel, G. Hammerl, C. Richter, and J. Mannhart, Appl. Phys. Lett. 89, 122101 (2006).

${ }^{20}$ C. Bell, S. Harashima, Y. Hikita, and H. Y. Hwang, Appl. Phys. Lett. 94, 222111 (2009).

${ }^{21}$ Y. Kozuka, M. Kim, C. Bell, B. G. Kim, Y. Hikita, and H. Y. Hwang, Nature 462, 487 (2009).

${ }^{22}$ M. Bibes, N. Reyren, E. Lesne, J.-M. George, C. Deranlot, S. Collin, A. Barthélémy, and H. Jaffrès, Philos. Trans. R. Soc., A 370, 4958 (2012).

${ }^{23}$ A. Zeilinger, J. Todt, C. Krywka, M. Müller, W. Ecker, B. Sartory, M. Meindlhumer, M. Stefenelli, R. Daniel, C. Mitterer, and J. Keckes, Sci. Rep. 6, 22670 (2016).

${ }^{24}$ D. W. Reagor and V. Y. Butko, Nat. Mater. 4, 593-596 (2005).

${ }^{25}$ F. Trier, G. E. D. K. Prawiroatmodjo, M. von Soosten, D. V. Christensen, T. S. Jespersen, Y. Z. Chen, and N. Pryds, Appl. Phys. Lett. 107, 191604 (2015).

${ }^{26}$ D. Fuchs, K. Wolff, R. Schäfer, R. Thelen, M. Le Tacon, and R. Schneider, AIP Adv. 7, 56410 (2017).

${ }^{27}$ G. Koster, B. L. Kropman, G. J. H. M. Rijnders, D. H. A. Blank, and H. Rogalla, Appl. Phys. Lett. 73, 2920 (1998).

${ }^{28}$ S. A. Chambers, M. H. Engelhard, V. Shutthanandan, Z. Zhu, T. C. Droubay, L. Qiao, P. V. Sushko, T. Feng, H. D. Lee, T. Gustafsson et al., Surf. Sci. Rep. 65, 317 (2010).

${ }^{29}$ S. J. Randolph, J. D. Fowlkes, and P. D. Rack, Crit. Rev. Solid State Mater. Sci. 31, 55 (2006).

${ }^{30}$ I. J. Luxmoore, I. M. Ross, A. G. Cullis, P. W. Fry, J. Orr, P. D. Buckle, and J. H. Jefferson, Thin Solid Films 515, 6791 (2007).

${ }^{31}$ D. Brunel, D. Troadec, D. Hourlier, D. Deresmes, M. Zdrojek, and T. Mélin, Microelectron. Eng. 88, 1569 (2011).

${ }^{32}$ Y. Z. Chen, D. V. Christensen, F. Trier, N. Pryds, A. Smith, and S. Linderoth, Appl. Surf. Sci. 258, 9242 (2012).

${ }^{33}$ X. Ke, S. Bals, A. R. Negreira, T. Hantschel, H. Bender, and G. Van Tendeloo, Ultramicroscopy 109, 1353 (2009). 